\title{
The Case for Multi-Tag RFID Systems*
}

\author{
Leonid Bolotnyy and Gabriel Robins \\ Department of Computer Science, University of Virginia \\ $\{l b 9 x k$, robins $\} @ c s . v i r g i n i a . e d u$
}

\begin{abstract}
Radio Frequency Identification (RFID) is a promising technology for automated non-line-of-sight object identification. However, factors such as object occlusions, metal / liquid opaqueness, environmental conditions, and radio noise degrade the overall availability, reliability, and dependability of RFID systems. We show that simply increasing the number of readers does not adequately address these issues. Instead, we propose tagging each object with multiple tags, and provide definitive experimental data showing that this strategy dramatically improves the effectiveness of RFID systems in the face of radio noise and other interfering factors. We solidify the case for multi-tag RFID systems by addressing obstacles to reliable object detection, and analyzing how multi-tags improve tag detection, even in the presence of (radio-opaque) metals and liquids. We discuss applications that will benefit considerably from multi-tags, and propose careful RFID system design through the deployment of appropriate types of multi-tags and anti-collision algorithms. We also analyze the economics of multi-tag RFID systems and argue that the benefits of multi-tags can substantially outweigh the costs in many current applications, and that this trend will become even more pronounced in the future.
\end{abstract}

Keywords: RFID, multi-tags, tag detection, tag receptivity, anti-collision

\section{Introduction to RFID}

A typical RFID system consists of readers (sometimes called beacons), tags (sometimes called transponders), and back-end servers that receive and process

\footnotetext{
*This research was supported by a Packard Foundation Fellowship, by NSF Young Investigator Award MIP-9457412, and by NSF grants CCR-9988331 and CCF-0429737. We thank Scott Krize for his help with the experiments. For additional related papers see http://www.cs.virginia.edu/robins
}

the information that the readers collect from the tags [3] [11] [13] [15] [16] [22] [25] [31] [38] [40]. There are three types of RFID tags: active, passive, and semipassive. Active tags have batteries on-board and can initiate transmission on their own. Passive and semipassive tags rely on power from a reader to engage in communication. Semi-passive tags have batteries onboard, but they are only used for on-board computations. Low-frequency $(125-135 \mathrm{kHz})$ tags can be read up to $30 \mathrm{~cm}$ away, high-frequency $(13.56 \mathrm{MHz})$ tags can be read up 1 meter away, and ultra high-frequency tags (868-915 MHz) can be read up to 7 meter away. On the other hand, active tags can be read more than 100 meters away [34]. There are two coupling mechanisms used by passive and semi-passive tags: inductive coupling and electromagnetic backscattering (or far-field propagation). In inductive coupling the reader creates a magnetic field between itself and the tags, which in turn derive power from this magnetic field. In far-field propagation, the reader sends a signal to a tag and the tag backscatters (i.e., reflects) a response back to the reader.

In many applications tags are passive in order to extend their useful lifetime and reduce the overall cost of an RFID system. Tag cost is currently a major obstacle for widespread deployment of RFID technology, and thus economic considerations tend to drive RFID system design [11]. Without suitable low-cost protocols for all of the functions of a tag, RFID technology may not be cost-justifiable for certain applications. Therefore, an important goal when designing RFID protocols is to ensure that they require the least amount of power and computational resources, while retaining their essential functionality. Some of the dominant applications that use RFID include supply chain management, inventory tracking, access control, library book checkout, cattle tracking, passport tagging, etc. The largest anticipated RFID deployment is the replacement of bar codes with RFID tags. For this deployment to be real- 
ized, the cost of an RFID tag must decrease substantially, into the low pennies range. Also, tag detection issues as well as privacy and security concerns need to be resolved in order to avoid commercial loses, as well as preempt the boycotting of RFID technology by privacy advocacy groups [1] [2]. We expect the work described here to help hasten the realization of full-scale commercial deployments of RFID technology.

\subsection{The Motivation for Multi-Tags}

Bar code scanners require a line-of-sight to the bar codes, and they usually have to be close to the objects being identified. Moreover, bar codes are scanned one at a time, and the bar code scanners (or the bar codes themselves) must physically move between successive reads. Such motion, being a mechanical process, limits the read rate to at best only a few bar codes per second. On the other hand, RFID readers can read hundreds of tags per second without requiring line-of-sight, thus allowing the easy automation of the reading process and making RFID-based identification very appealing commercially. As the identification process is automated, however, we must ensure the successful reading of all the tags within the readers' field.

Object detection is impeded by ubiquitous background radio noise. Moreover, metals and liquids reflect and/or absorb radio signals, further degrading the readers' ability to achieve accurate and complete tag identification. Missed items, even at a relatively low rate of $1 \%$, can result in large financial losses for stores with low profit margins that rely on RFID-enabled automatic checkout stations. This situation is real and serious, since milk, water, juices, and canned / metalfoil -wrapped (i.e., Faraday caged) goods are commonly stocked in markets. Practical experiments by WalMart in 2005 showed 90\% tag detection at case level, $95 \%$ tag detection on conveyor belts, and only $66 \%$ detection rate of individual items inside fully loaded pallets [18].

The report [39] by the Defense Logistics Agency showed that only $3 \%$ of the tags attached to objects moving through the Global Transportation Network (GTN) did not reach the destination (165 single-tagged objects were tracked in this study). However, the same report shows that only $20 \%$ of the tags were recorded in GTN at every checkpoint, and at one of the checkpoints fewer than $2 \%$ of tags of one particular type were detected. In addition, some of the tags were registered on arrival, but not on departure. As a result of these low object detection rates, accurate real-time tracking of objects moving through the GTN network was not possible. This report underscores the unreliability of object detection using a single RFID tag per object.

In addition to ambient radio noise, environmental conditions such as temperature and humidity can also adversely affect the success of object detection [14]. Moreover, objects moving at high speeds can have significantly reduced detection rates. The number of objects stacked together, variation in tag receptivity (even among tags from the same manufactured roll), and tag aging (and degradation in general) can diminish the detection probabilities as well. Objects tagged with a single tag are easy to steal (a simple metal foil placed over the tag can block detection). RFID systems used in healthcare pose a special dependability challenge, since RFID system deployment affects patients' welfare.

To address the problems discussed above, we propose attaching multiple RFID tags to each object, as opposed to using a single tag per object. Multi-tags will greatly improve object detection probability and increase reader-tag communication distance even in the presence of metallics, liquids, radio noise, and adverse environmental conditions. Multi-tags will greatly benefit theft deterrence and prevention applications, as well as dependable computing applications such as healthcare, where reliability, availability, and safety are required. All these benefits can be achieved at reasonable cost, as we discuss below.

\subsection{The Case for Multi-Tag RFID}

RFID technology is very appealing to many companies, but only a few large corporations such as WalMart and Best Buy have begun RFID trials and are considering near-term deployments. Meanwhile, other organizations are waiting to see the results of these trials before deciding on their own RFID strategies. This wait-and-see approach adopted by most companies is primarily due to the lack of concrete and convincing RFID business case studies. These companies view technological obstacles such as RFID system reliability, social discontent involving privacy rights, and the cost of RFID systems, as major potential impedements for wide-spread RFID deployment. Multi-tags can address many of these RFID deployment obstacles by improving object detection reliability, enhancing RFID security, and reducing the cost of RFID tags manufacturing (e.g., through economy-of-scale mechanisms). In short, multi-tag RFID may be exactly the enabling technology needed to help usher the RFID age into full bloom. 


\subsection{Organization of this Paper}

The rest of the paper is organized as follows. In Section 2 we discuss the optimal placement of multi-tags. In Section 3 we report on improvements in object detection using multi-tags, especially in the presence of metals and liquids. In Section 4 we show how the number of objects stacked together affects the average object detection probability, and in Section 5 we discuss the effect of tag variability and receptivity on object detection. Section 6 gives a number of examples where multi-tags can be very effective. In Section 7 we address and analyze the economics of multi-tags. Section 8 discusses the effect of multi-tags on tag interrogation algorithms, and we conclude in Section 9 with future research directions.

\section{Optimal Placement of Multi-Tags}

Our previous work on multi-tags has shown that object detection probability depends on the expected grazing angle of the radio signal from the reader to the tag [5]. Based on our analytical and experimental studies, if only one tag is used, can be positioned arbitrarily to maximize the average object detection probability. On the other hand, two tags should be positioned perpendicular to each other (i.e., in the $\mathrm{x}-\mathrm{y}$ and $x-z$ planes) in order to optimize the object detection probability. Similarly, for three tags, it is optimal to position the tags pair-wise perpendicularly (i.e., in the $\mathrm{x}-\mathrm{y}, \mathrm{x}-\mathrm{z}$, and $\mathrm{y}-\mathrm{z}$ planes). For four tags, it turns out that in order to maximize the expected signal incidence angle to at least one of the tags, it is best to position them parallel to the faces of a tetrahedron, a platonic solid. In cases when the goal is theft prevention and the number of tags per object is large, placement of multi-tags can be arbitrary, yet well-spread across the surface of an object in order to prevent the shielding of all of the tags.

Mathematical analysis and simulations have shown that the optimal positioning of multi-tags results in a double-digit increase in the expected grazing angle as the number of tags per object increases from one to two to three, but only a low single-digit improvement as the number of tags increases from three to four [5]. This suggests that adding an extra tag or two may dramatically improve object detection, but attaching the fourth tag to an object may not garner substantial detection probability improvement. These improvement trends are further corroborated by our experimental results with diverse object types [4].

\section{Object Detection with Multi-Tags}

To better understand our experimental results, we start by describing our RFID equipment and experimental setup. Then, we discuss our results involving metallic and liquid objects.

\subsection{Experimental Equipment and Setup}

Our experiments used commercial FCC-compliant equipment, namely Ultra High Frequency (UHF) readers from Alien Technology (model ALR-9800, four antennas, multi-protocol, $915 \mathrm{MHz}$ ) and ThingMagic (model Mercury 4). We utilized sets of linear and circular antennas from Alien Technology, and circular antennas from ThingMagic. A single Alien Technology reader antenna can either broadcast or receive signals, whereas the more versatile ThingMagic antenna can both send and receive signals. We used several types of tags from UPM Raflatac, the world's leading RFID tag manufacturer. In particular, we picked unipolar UPM Rafsec UHF "Impinj 34x54 ETSI/FCC" tags and bipolar UPM Rafsec UHF "Impinj 70x70 ETSI/FCC" tags for our experiments.

We performed the experiments in an otherwise empty room in order to minimize radio interference and reflection anomalies. We placed multiple tags on a diverse set of 20 solid non-metallic objects ${ }^{1}$ using four tags per object, and a set of 20 metal and liquidcontaining objects ${ }^{2}$ using three tags per object. We positioned tags perpendicular to each other whenever possible, and spread the tags as far apart in space across an object as possible, in order to minimize tag occlusions by other tags and/or objects. The experiments with solid non-metallic objects used sets of both unipolar and bipolar tags. The experiments containing metallic and liquid objects were performed only with unipolar UPM Rafsec UHF "Impinj 34x54 ETSI/FCC" tags.

We positioned Alien Technology reader antennas side-by-side in pairs, with each pair consisting of a sending and a receiving antenna. In the experiments described in this paper, the antennas were not intermixed (i.e., only sets of linear or sets of circular antennas were used at a time). Each pair of antennas was equidistant to the center of a plastic bag containing objects, placed 20.5 inches above the floor, and

\footnotetext{
${ }^{1}$ The multi-tagged solid non-metallic objects included common household items such as soap bars, cereal boxes, paper plates, plastic boxes, packaged foods, clothing items, etc.

${ }^{2}$ The multi-tagged metallic and liquid objects included cans of tomato sauce, canned vegetables, canned and bottled soda, bottled water, etc.
} 
aligned perpendicularly towards the center of the bag. The reader was operating in "inventory mode" using Gen-2 tag reading protocol. We allowed sufficient time for the reader to read all the tags within its range by performing many tag reads and maintaining adequate timeouts between reads to make sure that the effects of the environmental noise were minimized ${ }^{3}$.

In a separate set of experiments, circular ThingMagic antennas were equidistant and perpendicular to the bag containing the objects, located 33 inches above the floor, and in the rectangular "gate" formation. Each ThingMagic antenna was both sending and receiving signals. As with the Alien Technology hardware, we allowed sufficient reader time ${ }^{4}$ for object identification. We randomly (re)shuffled the tagged objects multiple times to change the tags' spatial orientations with respect to the reader's antennas, in order to improve the statistical significance of the results (the values reported in the tables and graphs below are averages over all random object shufflings). We also varied the power emitted by the antennas, keeping in mind that the distance at which tags can be detected is proportional to $\sqrt{\text { power. }}$

We wrote two computer software drivers to communicate with the two types of readers. The driver for the Alien Technology system utilizes API obtained from Alien Technology, whereas the driver for ThingMagic implements both the experiments' logic and the readercomputer communication protocol. Both drivers were implemented in Java. All reader-collected data was recorded for later analyses. Part of the tag-collected data processing was done in Java and part was done in Visual Basic for convenient data representation in graphical form using Microsoft Excel.

\subsection{Detection without Metals \& Liquids}

We briefly summarize here the average object detection results for solid non-metallic objects, as reported in [4], and then discuss object detection in the presence of metals and liquids. We performed the experiments for linear as well as for circular antennas using different readers, different tags, and varying power levels. The

\footnotetext{
${ }^{3}$ To enable others to reproduce our results, we specify here the Alien Gen-2 algorithm parameter settings used in our experiments: TAG_TYPE $=16$, ACQ_G2_CYCLES $=10$, ACQ_G2_COUNT $=100$, ACQ_G2_Q $=2$. Our source codes and scripts are available upon request.

${ }^{4}$ The internal algorithm of ThingMagic operates differently from that of Alien Technology. We allowed 2 to 4 seconds for the reader to identify all of the tags, depending on the number of objects.
}

results showed high double-digit improvements in average object detection probability of multi-tagged objects versus single-tagged ones with largest detection gains for 2-tagged and 3-tagged objects. The experiments confirmed our analyses regarding the importance of relative tag orientation. Interestingly, the orientation of a (unipolar) tag within its containing plane can also affect the detection probabilities. We also observed that multi-tags produce greater improvement in object detection than multiple readers, especially in systems with linear antennas.

\subsection{Detection with Metals \& Liquids}

Our prior multi-tag experiments were restricted to scenarios where the objects to be identified contained no metals or liquids [4]. In some practical scenarios, however, the items to be identified can contain mixtures of non-metallic objects, as well as metallic and liquid materials, making reliable object identification more problematic. It is more difficult to detect metallics and liquids because they tend to interfere with and occlude radio signals, thus preventing readers from receiving accurately decodable tag responses. Metallic and liquid objects can also occlude other nonmetallic objects and thus interfere with the detection of these as well.

When metals and liquids are present, the detection probabilities for solid and non-metallic objects decrease due to radio interference from the metallics and liquids. We observed a $4 \%$ to $10 \%$ decrease in the detection probability of solid objects, depending on the antenna type and the number of tags per object, as compared to situations where no liquids or metallics are present. Figure 1 shows the average object detection probability for solid objects for different antenna types and power levels. This data indicates an almost constant detection probability separation for linear antennas, and a narrowing detection probability gap for circular antennas, as the number of tags per object increases.

To detect metallic and liquid objects in our experiments, we had to considerably reduce the distance from the objects to the readers to ensure that tags are actually detectable at that range. Specifically, we reduced the approximate reader-to-tag distance to 32 inches, from the 55 inch range used for solid and non-metallic objects. In addition, we had to operate readers at high power levels only. To avoid using special tags that are specifically designed for metals and liquids, and to be able to compare relative improvements of multi-tags for solid/non-metallic objects with liquids 


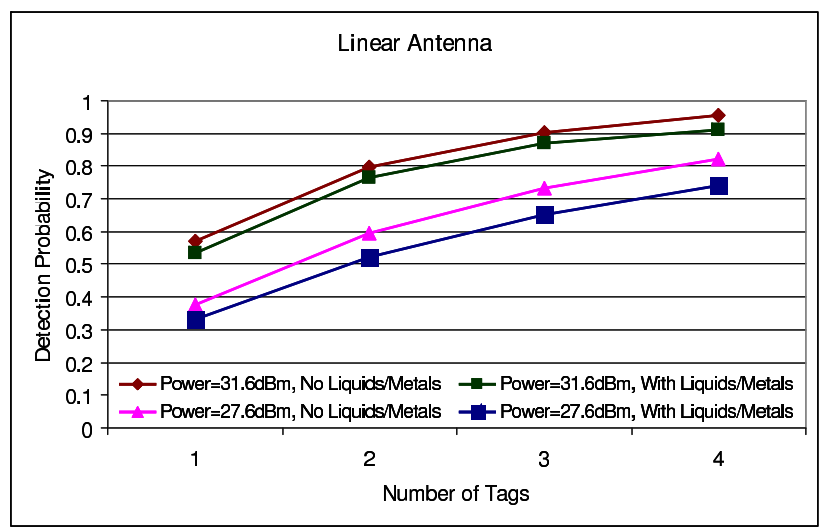

(a) Comparison of average detection probabilities using linear antennas for solid non-metalic objects when metallic/liquid objects are present and absent.

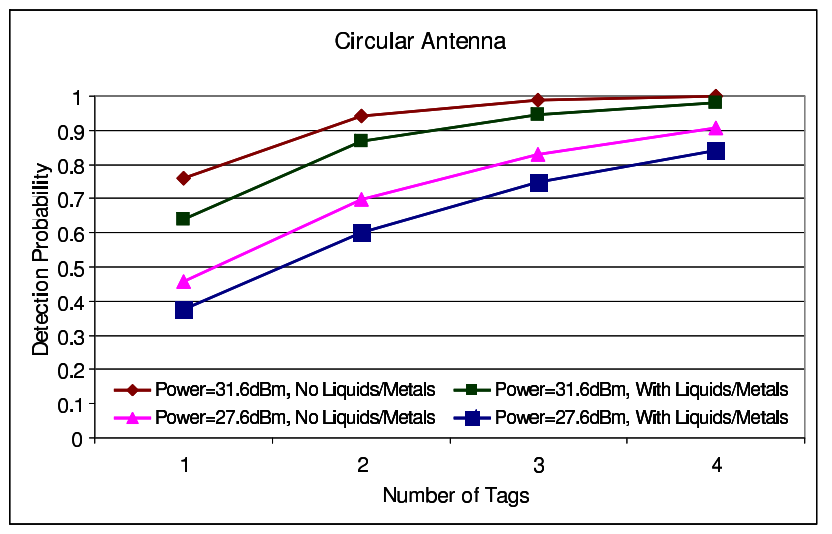

(b) Comparison of average detection probabilities using circular antennas for solid non-metalic objects when metallic/liquid objects are present and absent.

Figure 1. Comparison of average detection probabilities of solid non-metallic objects in environments that either include or exclude metals and liquids.

and metallics, we used a few millimeter thin spacers between the objects and the tags attached to them. This enabled bouncing radio signals to detect tags, yet kept the tags close enough to the metallic and liquid objects to retain the signal-interfering absorption and reflection characteristics of the liquids and metals.

Based on our experimental results, multi-tags are highly effective in improving object detection in the presence of metallics and liquids. We observed an almost linear improvement in metallic and liquid object detection when the number of tags per object is increased, as compared to the rapidly increasing and then leveling detection probability curve for solid nonmetallic objects. Figure 2 shows detection probability for several power levels and antenna configurations. Figure 3 shows the results of separate experiments performed using the ThingMagic hardware in order to determine how the detection probabilities of metallic and liquid objects vary for $1,2,3$, and 4 antennas. The results show rapidly vanishing improvements in object detection probabilities as the number of antennas increases, yet an almost linear improvement in object detection probabilities as the number of tags per object is increased, which is in line with our expectations.

\section{Effect of Object Quantity}

Aside from environmental conditions such as temperature and radio noise, and the presence of metallics and liquids in the objects' vicinity, the mere number of objects stacked together affects the average detec- tion probability of an object. This occurs because the objects to be identified act as radio signal occluders, shielding other objects' tags from the readers. To better understand the effect of the number of objects on the average object detection probability, we conducted several experiments. The results of these experiments confirmed our expectations and revealed interesting patterns that we describe next.

We performed two back-to-back experiments to determine the effect of the number of objects on the average object detection probability. In these experiments we used circular ThingMagic antennas and unipolar tags. In the first experiment, we grouped 15 solid non-metallic and 15 metallic and liquid objects and determined the average object detection probabilities for liquids and metallics, and separately for solid, nonmetallic objects. In the second experiment, we grouped 20 solid non-metallic and 20 liquid metallic objects, and again determined the average object detection probabilities. To ensure that the reader has sufficient time to detect all reader-visible tags in both experiments, we allocated 3 seconds for the reader to detect tags in the 15/15 experiment and (proportionally) 4 seconds for the 20/20 experiment. The detection probability statistics were calculated for various numbers of tags per object, as well as different numbers of reader antennas. For accurate comparison, in calculating the statistics in the second experiment we used a subset of 15 solid non-metallic and 15 liquid metallic objects that matched the objects in the first experiment. 


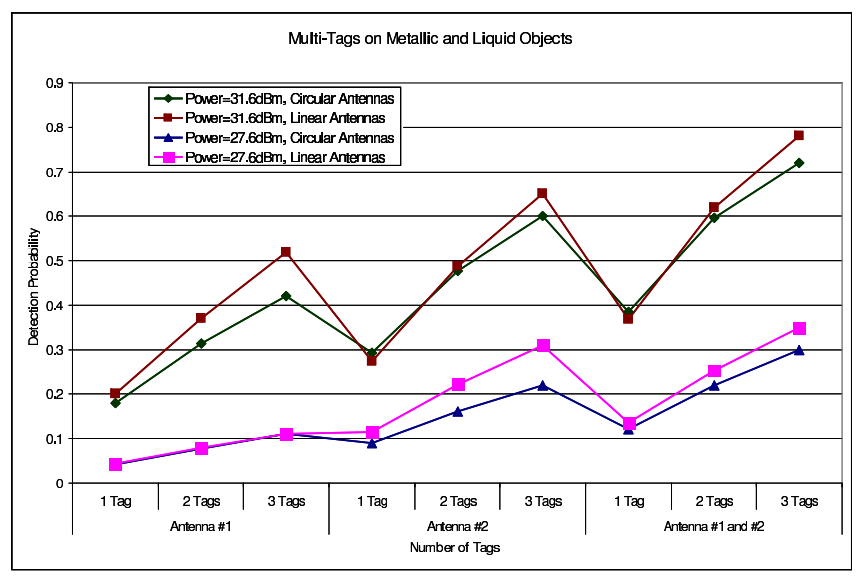

Figure 2. Comparison of average detection probabilities of metallic and liquid objects using one and two linear and circular antennas for various power levels.

We compared the average object detection probabilities between two experiments, varying the number of tags per object and the number of reader antennas. Figure 4 shows the results of this comparison for metallic and liquid objects. Observe that the average detection probability of an object in a $15 / 15$ experiment is greater than in a 20/20 experiment, as expected (since higher numbers of objects increase the likelihood of occlusions). The difference is more dramatic and vivid for metallic and liquid objects than for solid non-metallic ones because the reader is operating at a high power level in order to detect metallic and liquid objects.

Note that the difference in object detection probabilities between the two experiments is greater when more tags are attached to an object, and when multiple readers are used for object identification. This occurs due to an overall improvement in object detection when multi-tags and multiple readers are used. These experiments clearly illustrate that multi-tags have a more positive influence than multiple readers on detection probabilities, especially in the presence of metallics and liquids, and when identifying larger groups of objects.

\section{Tag Performance Variability}

Another factor that affects object detection is the intrinsic variability and receptivity of individual tags. It was observed that RFID tags from different chip manufacturers and various antenna geometries have distinct and varying detectability and receptivity performance [37]. The importance of tag receptivity and its use as a tag performance metric is addressed in [19]. Similarly,



Figure 3. Average detection probabilities of metallic and liquid objects using ThingMagic antennas, as the function of the number of tags per object and the number of reader antennas.

no two chips are truly identical due to inherent VLSI manufacturing variations [10]. In [4] we performed experiments to measure tag variability among "identical" UPM Rafsec UHF Impinj 34x54 ETSI/FCC tags that were shipped by the manufacturer on the same tag roll. Surprisingly, we found dramatic differences among seemingly identical tags, with up to an orderof-magnitude difference in detectability between the "best" and "worst" tags. These findings provide yet another incentive for deploying multi-tags in order to ensure more consistent object detection. Interestingly, tag variability can play a beneficial role in the construction of primitives that can be effectively used in RFID security and privacy algorithms [8].

\section{Applications of Multi-Tags}

Multi-tags can be deployed in a variety of useful applications and serve many purposes. They can be used for specific tasks such as determining the location and orientation of objects, as well as ensuring system reliability, availability, and even safety. In addition, multi-tags can be a considerable deterrent to illegal activities such as theft and forgery, and they can enhance RFID security and privacy. For example, multi-tags can speed up the execution of some algorithms through parallel computation. Below, we give examples of scenarios and systems where multi-tags can be effective. These examples do not cover all possible applications; rather, they serve mainly to illustrate the wide range of uses and applications of multi-tags. 


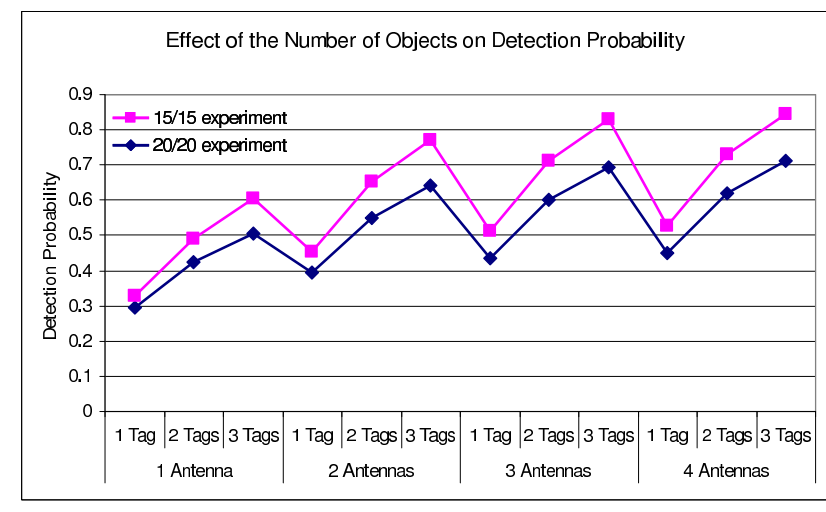

(a) The effect of the number of objects on the average detection probability.

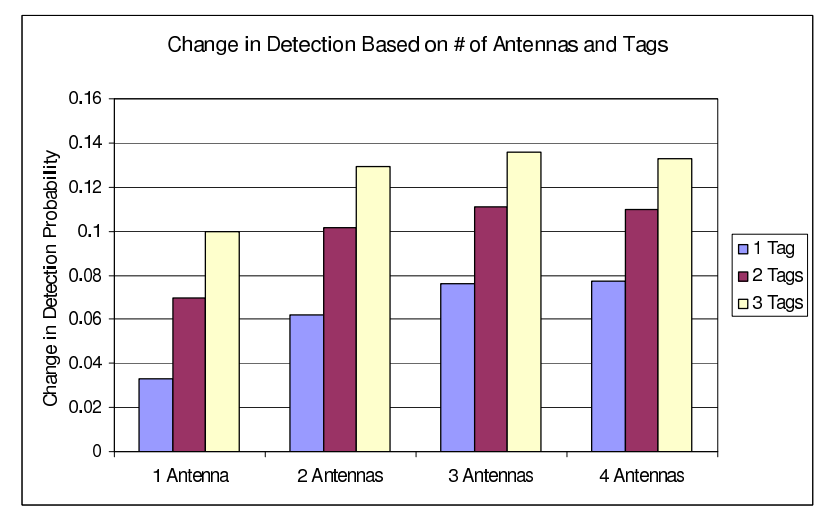

(b) The difference in average detection probability between the two experiments.

Figure 4. The effect of the number of objects on the average object detection probability. In the $15 / 15$ experiment we used 15 metallic and liquid objects, and 15 solid non-metallic objects. Similarly, in the 20/20 experiment, we used 20 metallic and liquid objects, and 20 solid non-metallic objects.

\subsection{Reliability}

There are many RFID applications where system reliability is critical. For example, in a store scenario, checkout RFID readers should reliably detect all items purchased by the consumer. Missed items, even at a relatively low rate of $1 \%$, can incur huge losses to lowprofit-margin businesses / stores. Also, objects moving through a supply chain should be detected reliably to enable accurate real-time inventory control and early theft detection. In general, in most applications where goods change hands or objects move through an RFID checkpoint, all objects should be detected and identified accurately. Multi-tags attached to objects will greatly increase objects' detection probabilities at a reasonable cost.

\subsection{Availability}

One example where multi-tags can improve system availability is in "yoking-proof" scenarios, where a potentially adversarial reader communicates with a group of tags and generates a proof that the tags were identified nearly simultaneously [7] [23]. The constructed proof is later verified by an off-line verifier. The integrity of the system hinges on the tags of all objects being detectable by the reader when required, since otherwise no valid proof can be created even by an honest reader. The problem is exacerbated because of the tight timing constraints of the protocol, and the inherent variations in tag receptivity [4]. In such "yokingproof" scenarios, multi-tags can be attached to each object, thus greatly increasing the probability of at least one tag per object being detectable. Note that here multi-tags may need to be physically connected to each other, so that they can consistently share their states with each other in order to prevent the possible forgery of a yoking proof (or else the tags must have distinct keys and the reader selects one detectable tag per object as a "leader" for that object).

Applications of yoking-proofs include verification by auditing bodies that a bottle of medicine was sold together with its usage instructions leaflet, or that safety caps were sold/delivered together with the associated devices, etc. Such scenarios can directly improve consumer safety by using multi-tags to ensure that a set of related objects is detected nearly-simultaneously. Another example of an application where availability is important is the real-time tracking of critical household or business objects such as remote controls, keys, firearms, and important documents, among others.

\subsection{Safety}

Another, perhaps unexpected, area where multi-tags can be of great benefit is safety. Specifically, multi-tags can be used in healthcare to track medical instruments (e.g., gauze sponges). For example, surgical sponges, among other foreign objects, are sometimes left inside humans during operations, causing highly undesirable consequences that adversely affect the patient. Recent medical studies [26] have shown surprisingly good results in detecting RFID equipped surgical gauze sponges during operations. However, to accurately de- 
tect all the sponges requires very careful and precise positionings of the reader. If the distance between the reader and the tags is increased even slightly, the tags may go undetected and thus possibly be inadvertently left inside the patient. In addition, the sponges may be located amid bodily liquids, further decreasing the detection probabilities. Finally, the tags on the sponges may break or malfunction, causing readers to miss tags, which may result in serious human injury.

Attaching multi-tags to surgical sponges will greatly increase the probability of all sponges being detected and accounted for, which would translate into improved patients' safety. Surgeons who participated in the study [26] estimated that the cost of RFID technology to detect sponges is about $\$ 144$ per patient. We believe that this cost can be substantially reduced, especially since such expenses can be amortized across many hospitals, operations, and patients. In addition, the cost of the RFID equipment deployed to ensure patient safety in hospitals may be viewed as part of the hospitals' insurance against malpractice lawsuits, and therefore this cost can be factored into the overall cost of a medical procedure or operation. Overall, we believe that investment in multi-tag RFID systems for safety-critical applications is highly cost-benefit justifiable. We discuss the economics of multi-tag RFID systems in greater detail in Section 7.

\subsection{Localization}

The location of a multi-tagged object can be more accurately determined than that of a single-tagged one. Well known location triangulation methods can be utilized to determine the position of each tag, thus reducing the error in computing a multi-tagged object's location coordinates. In addition, a carefully engineered multi-tag RFID system can be used to determine not only an object's position, but also its spatial orientation [17]. Directional antennas and orientationsensitive RFID tags can be deployed to make such a system highly effective. Creating a working prototype of such a system and applying it in real-world scenarios is an interesting area for future research.

\subsection{Packaging}

Many RFID tag types are delivered to the customer on a continuous paper roll, and the customer later programs the tags with unique IDs. We envision that tags will soon be cheap enough to embed into, e.g., adhesive packaging tape used to wrap packages and containers, thus simplifying the multi-tagging of boxed objects, and enabling automatic tag diversity and orientation selection to greatly improve object detection at negligible cost. With higher tag ubiquity and the multi-tagging of objects, the testing of RFID tags will be obviated, since even a low tag production yield will enable the overall system to function properly. The acceptability of lower tag manufacturing yields will further reduce the production costs, while ensuring high object detection probabilities as well as improved dependability and reliability of RFID systems.

\subsection{Security}

Multi-tags can be used to speed up the execution of private-key privacy-preserving authentication algorithms [6] [27], as well as provide a physical mechanism for resisting tag cloning [8]. In such algorithms, secret keys are assigned to the edges of a tree and tags correspond to tree leaves. The reader knows the secrets of the entire tree. The reader and a tag can authenticate each other by running a secure authentication algorithm for each edge of the tree of secrets, following a path from the root to the leaf where a tag is located. The secure authentication algorithm is keyed with the secret corresponding to the tree edge along the path. By arranging the tags at the leaves of the tree, the tag identification time is reduced from $O(n)$ to $O(\log (n))$ where $n$ is the total number of tags in the system. With multi-tags, such reader-tag authentication algorithms can run in parallel on different branches of a single tree level, as well as run predictively / speculatively at lower tree levels.

\subsection{Theft Prevention}

Another useful set of applications of multi-tags is in theft prevention. Increasing the number of tags attached to (or embedded in) an object will make it much more difficult for a thief to shield or remove all of the tags, thereby increasing the probability of getting caught. For example, one intriguing application of this could be the prevention of illegal deforestation by embedding tags in the trunks of living trees [5]. Since tags are very cheap compared to the cost of lumber (especially for rare or legally-protected trees such as Redwoods), the economics of such applications are financially viable. When logs are shipped and sold, they can be scanned for tags whose presence will determine the origin of the wood (and possibly convey other useful information, such as weather and environmental statistics tracked over the tree's lifetime). It 
would be prohibitively expensive for illegal loggers to detect and remove all the tags from a given tree trunk, thus substantially increasing the cost and risk of illegal deforestation, at a relatively low cost to the protection agencies.

DataDot Technology USA, Inc [42], produces "polyester substrate micro-dots" with laser etched identification data. These micro-dots can be applied to a surface, thereby marking it with unique identifiers that can later be read optically. A consumer applies micro-dots to his valuables and registers these microdots with DataDot Inc., which makes the information available to law enforcement agencies. DataDot Technology reports that this technology has greatly reduced the theft of marked items, and facilitated the recovery and return of stolen valuables [42]. We envision that RFID tags will eventually become cheap enough to enable the sprinkling of multi-tags onto objects similarly to "micro-dots", thus providing ubiquitous and permanent wireless identification capability. A thief can not realistically hope to reliably find and/or shield all of the numerous RFID tags thus sprinkled on an object (e.g., throughout a car). In addition, the attempted shielding of large collections of multi-tags can itself indicate a probable illegal intent.

Attaching the radio antenna(s) to the silicon chip, and the tag packaging itself incur the majority of the cost in RFID tag manufacturing [30]. However, if we use multi-tags for theft prevention as described above, we do not need to package the tags, nor be particularly precise or careful when attaching antenna(s) to chips. The mere large number of tags per object will guarantee that enough tags are still detectable, and thus deter theft. The simpler process of producing unpackaged tags will considerably streamline the tag manufacturing process and consequently reduce their cost. In addition, in such scenarios, manufacturing yields are no longer required to remain high, and tag testing may be skipped as well, further contributing to significant tag cost reductions. We discuss the economics of multi-tag RFID in more detail in the next section.

\subsection{Tagging Bulk Materials}

Cheap redundant multi-tags can be embedded into bulk materials (e.g., fertilizers, explosives, chemicals, propellants, crude oil, etc.) to prevent their unintended acquisition, transportation, and possible misuse. If tags are embedded into certain bulk materials at a reasonably small proportion to the size/quantity/weight of a substance, they will not adversely affect the nor- mal use of these materials (e.g., crude oil can be tagged at the rate of 10 multi-tags per barrel, and these tags can be removed during the final stages of the refinement process). If required, the tags can have limited lifespans or be (bio)degradable. The RFID tagging of fertilizers / explosives can help law enforcement agencies trace the producer and/or buyer. The tagging of bulk materials can also directly prevent criminals / terrorists from causing damage by enabling law enforcement agencies to detect the presence of dangerous substances in proximity (or ominously en route) to sensitive locations or particular sites of interest, hopefully before an illegal act transpires.

\section{The Economics of Multi-Tags}

Based on our object detection experiments [4] [5], it is clear that that object detection probabilities are far from perfect, even when multiple antennas and readers are used. Multi-tags, potentially in conjunction with multiple readers, can help address this problem. The cost of RFID tags in 2007 is around 10 U.S. cents a piece, making the multi-tagging of high cost items currently viable. In addition, the cost of tags is decreasing at an exponential rate following Moore's law, and this trend will enable the cost-effective tagging of even low-cost objects in the near future. Also, the cost of RFID tags is decreasing substantially faster than the cost of RFID readers, due to improving manufacturing yields and an economy-of-scale driven by massive deployments. Moreover, this price gap is expected to continue to widen due to the increasing demand for cheap RFID tags. The anticipated future omnipresence and ubiquity of RFID tags is expected to eventually reduce the cost of RFID tags into the sub-penny level.

\subsection{The Costs and Benefits of Multi-Tags}

The cost of passive RFID tags has been decreasing rapidly over the last decade. In 2001, the cost per tag was approximately $\$ 1.15$ when at least 500,000 units were purchased. For the same quantity, the tag cost in 2002 and in 2003 was $\$ 0.90$ and $\$ 0.50$, respectively [28]. In 2004 and in 2005, one could buy tags for $\$ 0.19$ and for $\$ 0.13$ a piece, respectively, in quantities of 1 million from Alien Technology [29]. In 2006, Avery Dennison announced that their tags cost $\$ 0.08$ in lots of 1 million or more [35]. Based on this historical data, we predict that tags will cost $\$ 0.06$ by the end of 2007 , and 5 cents in 2008. A 5 cent price point for tags was considered the threshold for supporting a strong business case for item-level tagging [36], and now this 
target is just around the corner. Based on the efforts of some companies and researchers working on RFID tag technology [30], we believe that $\sim 1$ penny tags will become a reality around the year 2011. Eventually, tags will be printed directly onto objects and cost less than a penny to produce. This cost milestone will make RFID a truly ubiquitous and affordable technology.

Figure 5 depicts the historical (and our projected) cost trends for passive tags (assuming 1 million tag lots). From this graph we can see that the cost of passive tags has been falling rapidly. The cost of readers has been decreasing slowly over the years (e.g., UHF RFID readers cost around $\$ 1,000$ in 2007). These cost trends suggest that readers, rather than passive tags, may be the cost bottleneck in deploying small RFID systems (i.e., with 100, 000 tags or fewer).

When considering the cost of RFID tags or even the cost of an entire RFID system, it is critical to also analyze the benefits that RFID brings to an application. A complete business analysis of deploying RFID should be performed, since the benefit of deploying RFID in an application can considerably outweigh the cost, even at today's prices. Specifically, the business analyses of RFID systems should take into account the direct savings that RFID deployment will enable, such as higher employee productivity, automated business processes, workforce reductions, and valuable information collected through RFID. In supply chain management scenarios the benefits of RFID deployment are tremendous. First, the merchandise can be tracked in real-time, allowing more efficient scheduling of operations. RFID may also allow reductions in the number of workers, since many currently manual processes can be automated. RFID can also prevent theft of goods, which are stolen predominantly by insiders ${ }^{5}$. Multitag technology enables objects to be tracked more effectively, not only during transport or check-out, but also during manufacturing and warehousing, which can significantly reduce theft and thereby increase profits.

\subsection{Tag Manufacturing Yield Issues}

Manufacturing yield is one of the main criteria that influence the cost of VLSI chips. This is because customers have to pay not only for the good chips delivered to them, but also for the defective chips that

\footnotetext{
${ }^{5}$ According to [12] [43], insider thiefs outnumber outsider thiefs six to one. It has been documented that over $1 \%$ of goods in retail stores are stolen [43], and the real losses due to theft are likely to be much higher, as companies tend to underreport theft statistics.
}

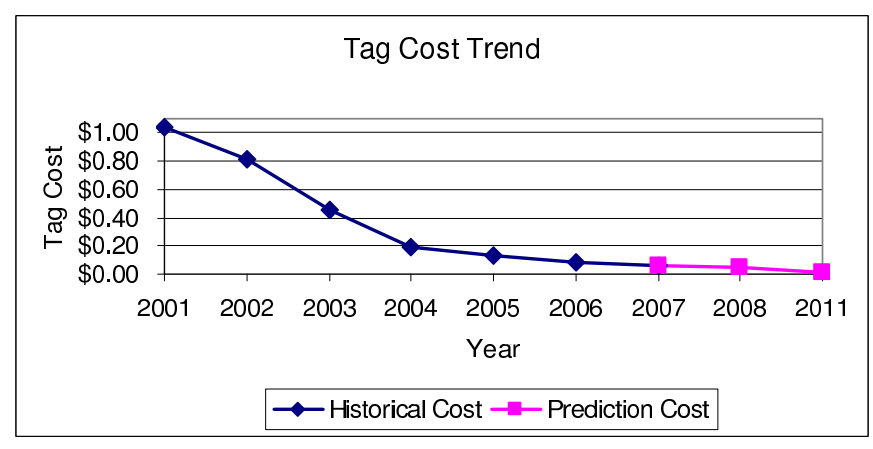

Figure 5. The cost trend of passive RFID tags over time, and our cost prediction for the future. The price per tag is based on the purchase of 1 million lots. We predict a 0.05 RFID tag price in 2008 , and a penny price point in 2011.

never made it out of the fabrication facility. For example, according to recent research by RFID vendors, as many as $30 \%$ of RFID chips are damaged during production when chips are attached to their antennas, and an additional 10 to 15 percent are damaged during the printing process [14]. Due to the redundancy built into multi-tag RFID systems, we can often ignore the manufacturing yield. Some manufactured RFID tags may be defective, while others may fail in the field, but if multiple tags are attached to each object, the probability that all the tags fail is still quite small. This considerably increases the overall reliability of a multitag RFID system, and decreases the tag manufacturing costs (e.g., expensive manufacturing steps such as testing may be dispensed with).

The failure rate of deployed RFID tags in the field is estimated to be as high as 20\% [32]. This large failure rate induces an additional cost pressure on RFID tag manufacturing, since individual tags must be made more reliable, and/or extensively tested after manufacturing. Even after packaging, tags may become defective. For example, $5 \%$ of the tags that we purchased for our experiments were marked by the manufacturer as defective; moreover, we discovered several additional inoperable tags during the tag programming phase of our experiments. As with the yield issue, multi-tags allow us to ignore damaged tags and statistically rely on the promise that enough multi-tags will remain operational to satisfy an application's requirements. This property of multi-tag systems helps to improve the overall reliability and cost of deployed multi-tag RFID systems. 


\subsection{RFID Demand Drivers}

A strong driver of cost in RFID systems is the scope of the demand for this technology. With increases in demand, the number of produced RFID units will increase, which drives the amortized development costs down. However, many companies are hesitant to deploy RFID technology because the business case is not entirely clear and/or proven. This classic "chicken and egg" dilemma has inhibited the massive deployments of RFID systems. With improvements in RFID technology, the cost of RFID systems should decrease, creating a more convincing business case for companies and accelerating the demand for the technology, which will in turn reduce the amortized cost of RFID tags even further. The demand for RFID will be driven by many companies with a wide range of specializations and fields, led by major players such as Wal-Mart, and the desire to remain competitive in rapidly evolving marketplaces. Consequently, companies will experience mounting pressures to adopt RFID technology, and multi-tag -based strategies will help bootstrap undecided companies into this technology and help propel them into the RFID age.

\subsection{Cost Effective Tag Design Techniques}

Overall tag cost can be reduced by developing better and cheaper tag components and assembling them in a more cost-effective manner. Below we give some practical examples of advanced memory design, antenna design, and assembly technologies to illustrate how technological developments drive down RFID costs.

Memory Design: The cost of RFID tags can be reduced through innovative lower-cost memory design technologies. For example, the chip manufacturer Impinj Inc, uses "self-adaptive silicon", which enables the low-cost reliable analog storage of bits in floating gates [20]. This allows memory design with no additional photo masks or other steps used to create non-volatile EEPROM, yet the floating gates reliably store memory even during memory updates. The result is a much simpler memory technology that adjusts itself during its operational lifetime, thus minimizing the impact of aging, voltage and temperature fluctuations on the reliability of memory storage [20].

Assembly Technology: Another way to decrease the tag cost is to speed up the tag manufacturing and packaging processes. For example, Alien Technology has developed "Fluidic Self Assembly" (FSA), which allows placement of a large number of very small com- ponents across the surface in a single operation, significantly speeding up tag assembly. This technology involves flowing tiny microchips in a special fluid over a base containing holes shaped to catch the chips [21]. While this self-assembly process involves some randomness, most interconnections are established correctly, thereby reducing the assembly cost. FSA is able to package up to 2 million RFID chips per hour, compared to only 10 thousand chips per hour possible using conventional technologies, cutting the chip manufacturing cost by approximately $50 \%$ [21]. In addition to Fluidic Self Assembly, Alien Technology uses High Speed Strap Attach Machines (HiSAM) for very fast packaging of FSA straps. Each HiSAM machine can produce 250 million tags per year, which allows for very fast high volume production, and corresponding drastic reductions in manufacturing costs.

Antenna Design: In addition to designing antennas with improved receptivity and orientation, measures can be taken to lower antenna costs. For example, Symbol Technologies reduced the cost of antennas by manufacturing them from aluminum rather than silver. The company also compressed antennas into small, low-powered inlay, thus reducing tag area and cost. In addition, their "charge pump" technology improves tag power efficiency and enables greater tag detection range [41].

The two British companies Carclo and Xennia Technology formed a partnership to design printable antennas, rather than etching them, which reduces antenna cost. In addition, the companies' printers can print very thin (half-micron) antennas onto paper and cardboard, thus making the antennas recyclable. Antennas can also be printed over the chip itself, strengthening the bond between the chip and antenna [33].

\subsection{Summary of Multi-Tag Economics}

RFID technology leverages Moore's Law in the positive direction. RFID tags are getting both smaller and cheaper over time, resulting in a multiplicative corresponding reduction in tag cost. In addition, RFID tag yields are improving, further compounding the effect of these trends on cost reduction. Also, engineering and manufacturing tolerances for RFID chips are much larger than for high-end chips (e.g., RFID chips can operate at low clock speeds, extreme miniaturization is not a prominent problem in RFID production, etc.). Moreover, the VLSI manufacturing equipment for RFID tags does not have to be cutting-edge, which 
reduces the cost pressure when constructing tag fabrication facilities. Rapidly increasing demand for RFID, along with cheaper manufacturing techniques and improving yields, is expected to rapidly bring the cost of RFID tags into the sub-penny levels in the near future, making multi-tags ever more affordable.

In short, multi-tags are absolutely economically viable, and their benefits are bound to become even more dramatic over time.

\section{Effect on Anti-Collision Algorithms}

Anti-Collision algorithms enable a reader to uniquely identify tags while minimizing the number of tag broadcasting collisions (i.e., simultaneous interfering transmissions by the tags). Multi-tags have no effect on two variants of Binary Tree-Walking [11] [24], and may at most double/triple the total read time for dual/triple-tags over single tags for Slotted Aloha [11] and for Randomized Tree-Walking [6] [9] [44]. Our theoretical and experimental study of multi-tags addressed how multi-tags improve object detection [4] [5]. It is worth noting, however, that since not all tags are detected, the time required to identify all reader-visible tags is considerably less than double (or triple) the time needed to identify single-tagged objects.

In particular, from our experiments we observed that $25 \%$ to $75 \%$ of all tags on solid/non-metallic objects are detected with one reader antenna, depending on its type and power level. The percentages are much lower for metallic and liquid objects. Therefore, attaching two tags to each object may not add any significant overall time delay for object identification. Moreover, current RFID technology can read hundreds of tags per second, making the increase in the number of tags insignificant, even in real-time systems. Finally, in many scenarios the benefits of successfully identifying all the objects certainly justifies a modest increase in identification time. Based on the above observations, RFID system designers should select an appropriate anti-collision algorithm based on the number of objects that may have to be identified nearly simultaneously, the number of tags attached to each object, and the expected objects' velocities (if the objects to be identified are not stationary).

\section{Conclusion}

There are many obstacles to reliable RFID-based object identification. Environmental conditions such as temperature and humidity, ambient radio noise, and object geometries and occlusions can significantly interfere with object detection and identification. Dramatic variations in tag receptivity and detectability, even among tags of the same type and production batch, reduce the reliability of tag detection. The metals and liquids present in or around objects (or the environment) can reflect or absorb radio signals, thus preventing accurate signal decoding. In addition, the object density, concentration, and placement geometry can adversely affect object detection.

Our experiments showed that multiple readers improve object detection only moderately, yet multi-tags provide much more dramatic gains in average object detection probability. We observed that multi-tags are very effective in dealing with radio noise, tag variability, and the presence of metallics and liquids among objects, as well as high object densities. We gave examples of numerous applications that could greatly benefit from multi-tags. We analyzed the economics of multi-tags and argued that multi-tags are costeffective even today for many cost-sensitive, safetycritical, and security-oriented applications. We predicted that multi-tags will become cost-justifiable for many more applications in the near future, as the cost of passive tags continues to rapidly drop. We also stressed the importance of careful RFID system design to ensure the desired operation and performance.

In summary, we believe that multi-tag RFID technology promises many benefits to numerous applications, and will expedite reductions in tag manufacturing cost. This will positively tip the cost-benefit scale in favor of massive RFID deployments, and encourage many companies, organizations, and communities to join the age of ubiquitous RFID.

\section{References}

[1] K. Albrecht and L. McIntyre. Spychips: How Major Corporations and Government Plan to Track Your Every Purchase and Watch Your Every Move. Plume, 2006.

[2] K. Albrecht and L. McIntyre. The Spychips Threat: Why Christians Should Resist RFID and Electronic Surveillance. Nelson Current, 2006.

[3] M. Bhuptani and S. Moradpour. RFID Field Guide - Deploying Radio Frequency Identification Systems. Sun Microsystems Press, New Jersey, 2005.

[4] L. Bolotnyy, S. Krize, and G. Robins. The practicality of multi-tag rfid systems. In Proc. International Workshop on RFID Technology - Concepts, Applications, Challenges (IWRT 2007), Madeira, Portugal, June 2007. 
[5] L. Bolotnyy and G. Robins. Multi-tag radio frequency identification systems. In Proc. IEEE Workshop on Automatic Identification Advanced Technologies (Auto-ID), pages 8388, October 2005.

[6] L. Bolotnyy and G. Robins. Randomized pseudo-random function tree walking algorithm for secure radio-frequency identification. In Proc. IEEE Workshop on Automatic Identification Advanced Technologies (Auto-ID), pages 43-48, October 2005.

[7] L. Bolotnyy and G. Robins. Generalized 'yoking proofs' for a group of radio frequency identification tags. In International Conference on Mobile and Ubiquitous Systems (Mobiquitous), San Jose, CA, July 2006.

[8] L. Bolotnyy and G. Robins. Physically unclonable function -based security and privacy in rfid systems. In Proc. IEEE International Conference on Pervasive Computing and Communications (PerCom 2007), New York, March 2007.

[9] Auto-ID Center, 2003. Draft Protocol Specification for a $900 \mathrm{MHz}$ Class 0 Radio Frequency Identification Tag.

[10] Y. Chen, A. B. Kahng, G. Robins, and A. Zelikovsky. Area fill synthesis for uniform layout density. IEEE Trans. Computer-Aided Design, 21(10):1132-1147, October 2002.

[11] K. Finkenzeller. RFID Handbook. Wiley \& Sons, 2003.

[12] National Association for Shoplifting Prevention (NASP). Shoplifting statistics. www.shopliftingprevention.org.

[13] A. Furness and I. G. Smith. RFID Compendium - The Technology and Where to Use It. Auto ID Service Providers, Halifax, England, 2004.

[14] Gao. Key considerations related to federal implementation of radio frequency identification technology. http://www.gao.gov/new.items/d05849t.pdf, June 2005. Testimony Before the Subcommittee on Economic Security, Infrastructure Protection, and Cybersecurity, House Committee on Homeland Security.

[15] S. Garfinkel and B. Rosenberg. RFID - Applications, Security, and Privacy. Addison-Wesley, 2006.

[16] C. Heinrich. RFID and Beyond. Wiley Publishing, Indianapolis, IN, 2005.

[17] S. Hinske. Determining the position and orientation of multi-tagged objects using rfid technology. In Fifth Annual IEEE International Conference on Pervasive Computing and Communications Workshops (PerComW'07), pages 377-381, White Plains, NY, March 2007.

[18] IDTechEx. Rfid progress at wal-mart. www.idtechex.com/products/en/articles/00000161.asp, October 2005.

[19] Impinj. Receptivity - a tag performance metric. www.impinj.com/files/MR_MZ_WP_00005_TagReceptivity.pdf, December 2005.

[20] Inc. Impinj. Our technology. http://www.impinj.com/ advantage/our-technology.aspx.

[21] Alien Technology Inc. Fsa manufacturing. www.alientechnology.com/technology/fsa_manufacturing.php.

[22] N. Jilovec. EDI, UCCnet and RFID - Synchronizing the Supply Chain. 29th Street Press, Loveland, CO, 2004.

[23] A. Juels. 'yoking-proofs' for rfid tags. In R. Sandhu and R. Thomas, editors, International Workshop on Pervasive Computing and Communication Security, pages 138-143, Orlando, FL, USA, March 2004.
[24] A. Juels, R. Rivest, and M. Szedlo. The blocker tag: Selective blocking of rfid tags for consumer privacy. In V. Atluri, editor, Proc. ACM Conference on Computer and Communications Security, pages 103-111, Washington, DC, USA, October 2003.

[25] R. A. Kleist, T. A. Chapman, D. A. Sakai, and B. S. Jarvis. RFID Labeling - Smart Labeling Concepts \& Applications for the Consumer Packaged Goods Supply Chain. Printronix, Inc., Irvine, CA, 2004.

[26] A. Macario, D. Morris, and S. Morris. Initial clinical evaluation of a handheld device for detecting retained surgical gauze sponges using radiofrequency identification technology. Arch Surg, 141:659-662, 2006.

[27] D. Molnar and D. Wagner. Privacy and security in library rfid issues, practices, and architecture. In Proc. ACM Conference on Computer and Communications Security, pages 210-219, Washington, DC, USA, October 2004.

[28] R. Moscatiello. Forecasting the unit cost of rfid tags. http://www.mountainviewsystems.net/ Forecasting

[29] M. O'Connor. Alien drops tag price to 12.9 cents. http://www.rfidjournal.com/article/articleview/1870/1/1/, September 2005. RFIDJournal.

[30] P. Peumans. Monolithic, low-cost rfid tags. http://peumans-pc.stanford.edu/research/monolithiclow-cost-rfid-tags, 2006. Project at Stanford Organic Electronics Lab.

[31] T. Polizzi. RFID in the Enterprise for Cross-Enterprise Business Automation. WCCN Publishing, Irvine, CA, 2004.

[32] RFIDJournal. Rfid system components and costs. www.rfidjournal.com/article/articleview/1336/3/129/.

[33] RFIDJournal. New way to print ink antennas. New Way to Print Ink Antennas, October 2003.

[34] M. Rieback, B. Crispo, and A. Tanenbaum. The evolution of rfid security. In Pervasive Computing, January-March 2006.

[35] M. Roberti. A 5-cent breakthrough. www.rfidjournal.com/article/articleview/2295/1/128/, May 2006. RFIDJournal.

[36] S. Sarma. Towards the five-cent tag. Technical Report MIT-AUTOID-WH-006, 2001. Auto-ID Labs.

[37] E. Schuster, T. Scharfeld, P. Kar, D. Brock, and S. Allen. Analyzing the rfid tag read rate issue. http://mitdatacenter.org/CutterITAdvisor.pdf.

[38] S. Shepard. RFID - Radio Frequency Identification. McGraw-Hill, New York, 2005.

[39] Inc. SRA International. Resolute ordinance movement evaluation. http://www.dla.mil/j-6/ait/ Documents/Reports/Resolute_Ordinance_May2001.aspx, May 2001. Appendix F - GTN Tag History.

[40] P. J. Sweeney. RFID for Dummies. Wiley Publishing, New Jersey, 2005.

[41] Symbol Technologies. Rfid tags. http://www.symbol.com/ products/rfid-readers/rfid-tags.

[42] DataDot Technology. http://www.datadotusa.com/.

[43] M. Vargas. Shoplifting and employee theft recovery up last year. http://retailindustry.about.com/od/lp/a/ bl_hayes_theft.htm. From 17th Annual Retail Theft Survey by Jack L. Hayes International, Inc.

[44] S. Weis. Security and privacy in radio-frequency identification devices. Master's thesis, MIT, May 2003. 